ISSN: 2277-3754

ISO 9001:2008 Certified

International Journal of Engineering and Innovative Technology (IJEIT)

Volume 11, Issue 4, October 2021

\title{
Study of the STBC based MIMO System Modeling and Evaluation
}

\author{
Kawandal Jayswal, Pankaj Vyas \\ Department of Electronics Communication, SISTec, Bhopal (M.P.)
}

Abstract: There are many applications using the small MIMO antenna structure for performance improvement. The Long Term Evolution (LTE) and Wi-Fi wireless modems are good example of the MIMO based modeling. Many coding techniques have been designed for enhancing the performance of the MIMO channels. This paper has presented the comprehensive study of the Coding techniques used for MIMO system specially using the Space Time Block Codes $(S T B C)$. Basically the coding techniques are used for improving the estimation of the MIMO Channel states information (CSI). Various design challenges of the channel estimation for wireless communication are addressed and problems are identified. The comparison of various SCBC based MIMO channel estimation methods have been tabulated based on the BER and MSE performances.

Keywords: - Wi-Fi, MIMO, STBC Coding, Channel Estimation, BER, Capacity, Rayleigh Fading.

\section{INTRODUCTION}

Much research has been done to improve the transmission and performance capacity of the wireless communication system. A standard wireless communication system uses a single point to identify a channel link. To meet the need for high data transfer over time, Multi-input, Multi-Output (MIMO) systems are popular. An example of the basic communication channel and MIMO structure is shown in Figure 1. The $\mathrm{X}$ vector represents the input signal vector on the transmitters and the $\mathrm{Y}$ represents the vector at the receiver. The MIMO system provides $\mathrm{MxN}$ horns mounted on a space-varying structure.

In the MIMO system the signals corresponding to every transmitting and receiving antenna are represented respectively as;

$$
X=x_{1}, x_{2} \cdots \cdots x_{M} \text { and } Y=y_{1}, y_{2_{s}} \cdots \cdots y_{N}
$$

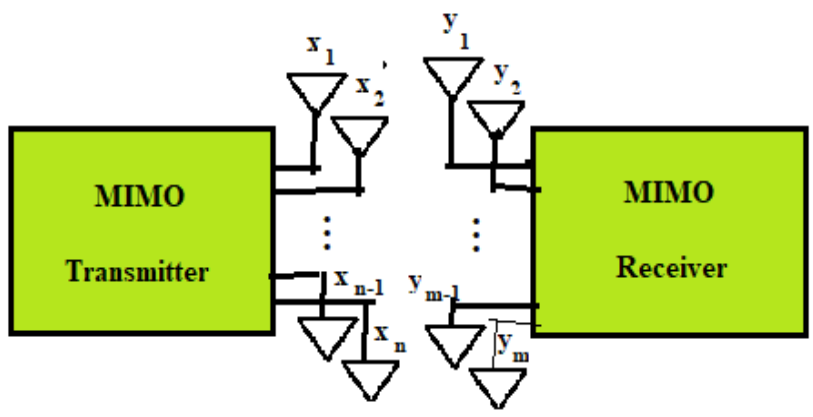

Fig. 1. The basic MIMO channel structure TX/RX

Manuscript received: 22 September 2021

Manuscript received in revised form: 20 October 2021

Manuscript accepted: 6 November 2021

Manuscript Available online: 15 November 2021
One of the key applications of the MIMO system is the updated LTE 3G UMTS form to meet the needs of the most demanding customers. An example of critical customer demand is fast data and high quality $(\mathrm{QoS})$ service. There are many coding methods designed to meet the best QoS especially in static management systems. The LTE system uses the MIMO antenna structure with Orthogonal Frequency Division Multiple (OFDM). LTEMIMO offering $100 \mathrm{Mbps}$ via 2x2 MIMO channel for $20 \mathrm{MHz}$ systems. It is therefore very necessary to design the first methods of good writing in order to obtain the best error rates in MIMO systems. Acceptance of MIMO killings is highly dependent on the direct measurement of Channel State Information (CSI). MIMO channel details say they are transmitted randomly and are timedependent. The channel is therefore made using the Rayleigh fading system model.

This paper has identified different limitations of the MIMO channel framework using encoding methods. The challenge of MIMO design includes pilot channel usage limitations and blind limitations for channel and channel codes, power measurements with channel configuration techniques including Least Square Error (LSE) and Minimum Mean Square Error (MMSE) CSI power rating.

The system with MIMO is used to improve the power of the wireless channel. It has become apparent that there is more information flow on overcrowding in wireless communication channels. Especially during the COVID epidemic the flow of digital data has increased dramatically. The Wireless Wi-Fi network access is a great way to work online in the current environment. Alamouiti based space time coding methods [1-5] are widely used to improve the efficiency of MIMO wireless channels. Methods of the time constructed space blocks (STBC) used in the orthogonal space used for the above mentioned purpose.

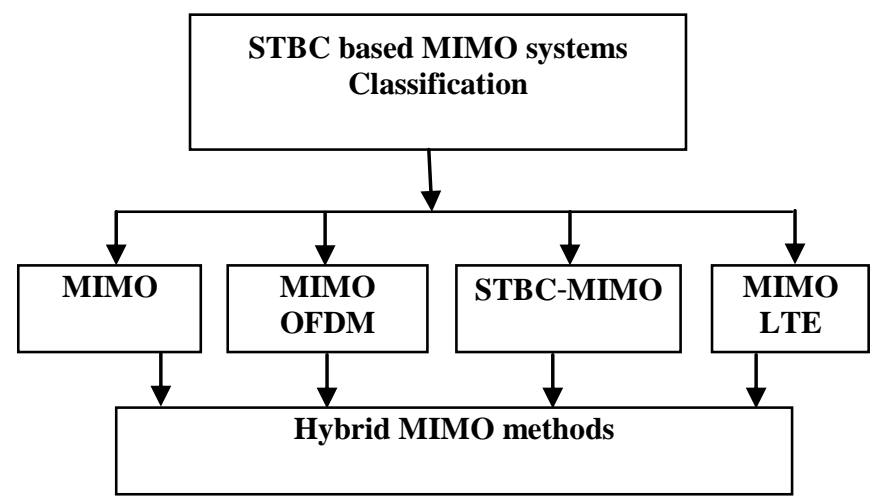

Fig.2. Classification of MIMO based on STBC code 
ISSN: 2277-3754

ISO 9001:2008 Certified

International Journal of Engineering and Innovative Technology (IJEIT)

Volume 11, Issue 4, October 2021

The MIMO system broad classification are shown in the Figure 2, It can be seen from the Figure 2 that, STBC based MIMO systems designs are broadly classified as basic MIMO, MIMO-OFDM systems, MIMO-STBC and the MIMO-LTE frequently being opted the STBC based coding methods.

The structure of STBC coding based MIMO wireless Wi-Fi communication system is shown in Figure 3. There is a STBC encoder and decoder for improving the efficiency of data transmission over MIMO channels $\mathrm{H}$.

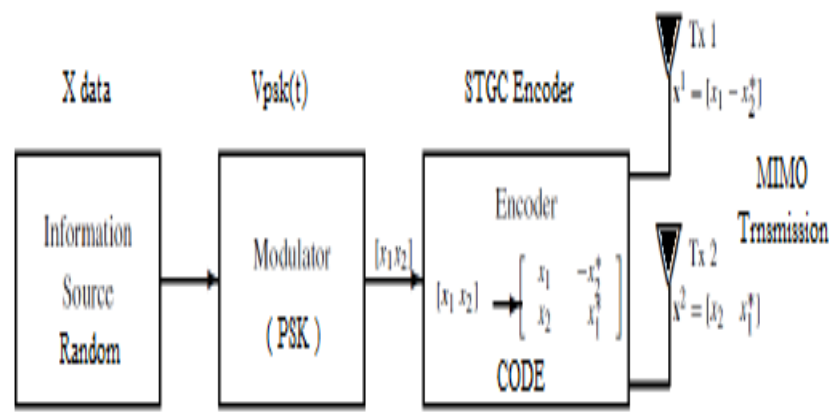

Fig.3: MIMO-STBC based encoder for communication system

$N_{t}$ is number of transmitting side antennas, $N_{r}$ is receiving side antennas, let the $X$ is signal transmitted, $\mathrm{N}$ is the zero mean Gaussian noise.

\section{REVIEW OF LITERATURE}

Various types of deviation modifications in MIMO WiFi for LTE and OFDM systems are presented here. Exploration activities include a variety of channel measurement methods such as pilot based, daze channel, LMS and RLS, LS and MMSE and other channel measurement techniques..

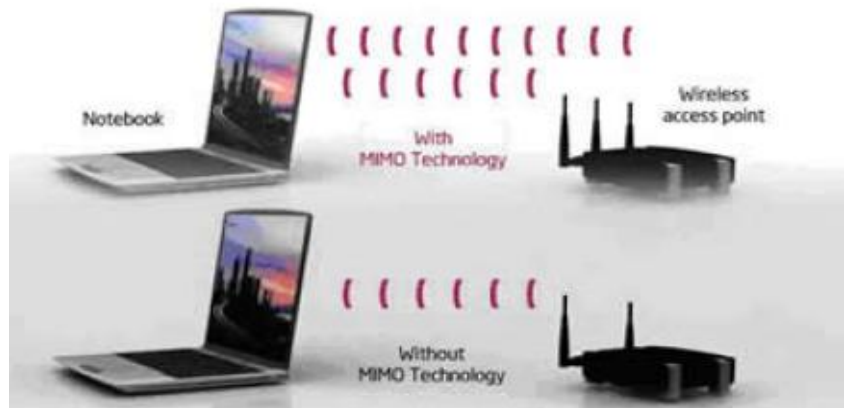

Fig. 4. An example of MIMO wi-fi hot spot application

S Nandi [1] has presented the evolution of the OFDM system using the MIMO systems for numerous MIMO transceivers using the Alamouti STBC coding techniques. They have plotted the theoretical vs simulated bit error rates BER for the STCB block codes using the binary BPSK modulation techniques.

Authors in [2, and 3] showed great enthusiasm for a variety of topics including MIMO systems, finding MIMO channel capacity, encoding MIMO channel, space-time coding. S. Karri et al. [4] has presented the good study of the MIMO based WiMax systems based on usng the Orthogonal STBC (OSTBC) coding method Theoretical BER of system is compared to MRC (Maxima ratio based combiner) and the proposed approach.

Jyoti P. Patra et. al. [5] "STBC based on Site Assistant channel based on OFDM System in accordance with the Selected Frequency Channel" "multiplexing division (OFDM) that evaluates multipath shading channels. Channel ratings are based on a driver-type game system that includes passengers. The placement was done simultaneously on each of the OFDM image segments and intersects between radio transmissions due to the benefits of the carrier carrying carrier, the actual transmission flags fully recovering from the integrated apartment. There is a small number to check the station that the passenger of the vehicles under the driver while the suspension is complete for the person in charge of the details. The development of a channel measurement strategy is not in line with OFDM's optical input system (SISO) in terms of image error rate (SER). The reemergence comes from the indication that STBC OFDM offers the best.

Manisha Patel et al. [6] suggested a planned release of the pilot assistant MIMO-STBC based channel estimation system with a analogous time varying channel. They created a MIMO novel rating to embrace the pilot's image and help with vulnerability which keeps it usable for even bit blurring channels. A great opportunity for the pilot image embedded in the information stream posted by the orthogonal STBC encoder. In the reception area, they created an important process for measuring the previous MIMO channel that will be used with the STBC decoder. An accurate and accurate PSAM test strategy is expected by MIMO based on orthogonal STBC codes.

Mohamed Marey, et. al [7] "Dazzle STBC Identification for Multiple-Antenna (MA) OFDM Systems" A time zone release that separates the evidence of various orthogonal recultrent division multiplexing (FDM) P. K. M. Ho, et al [9] have presented the inpurgact CSI realization for MIMO system design. Pankaj kumar bhart has preeted the MIMO-OFDM design using the STBC coding and evaluated the BER using te MRC combining.

Aws Amer et al [11] in there paper investigated a STBC coding system for simulation of MIMO-OFDM in the MATLAB. The performance is analyzed based on known and unknown Information's of the states of channel (CSI). The channel was modeled using the STBC coding strategies for the Rayleigh fading channel. They presented the good mathematical modeling for STBC based MIMI system design for fading channel to represent the CSI.

An example representation of the MIMO system consideration for the STBC coding based system is given in the Figure 5. 
ISSN: 2277-3754

ISO 9001:2008 Certified

International Journal of Engineering and Innovative Technology (IJEIT)

Volume 11, Issue 4, October 2021
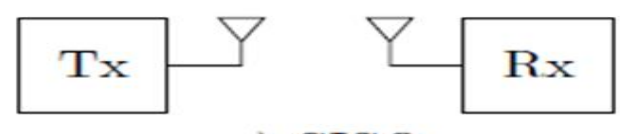

a) SISO
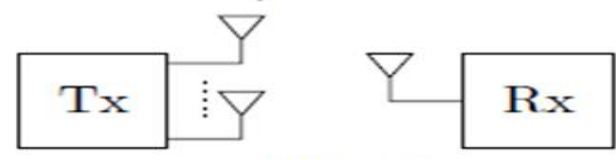

b) MISO

Fig. 5. The MIMO system consideration for the STBC based system

MIMO Design Challenges

One of the most difficult challenges facing wireless engineers and developers is the bottle system introduced by a wireless link layer. Finding high levels of data on a wireless channel is a major problem for many reasons. Wireless channel is a bad time to distribute time. Signal sent to wireless channel directed to Multi patch modes:

The main reasons for the construction of the MIMO system are as follows

- MIMO antenna systems are designed to overcome the effect of low penetration.

- The need for high-speed data transmission over longdistance communication is one of the main reasons for the emergence of a multidisciplinary input system.

- Multiple input antenna and multiple output provides high data throughput, as well as high reliability with the same bandwidth as a single input and output system (SISO).

- It also provides high bandwidth and reliability without the need for additional bandwidth.

- Increasing the data transfer capacity over a limited channel is beneficial for designing a multi-threaded MIMO system.

- It is a big challenge to design the system with the use of the space tie codes for performance enhancement.

\section{ORTHOGONAL SPACE TIME BLOCK CODING}

The space time coding (STC) based, detection method is used here for enhancing reliability of MIMO channel and error performance. In addition, spatial division multiplexing (SDM) is used to maintain fast data rate for wireless communication without excess transmission power and bandwidth scope. The STC Encoding in block basis is capable of handling the diversity in space and time. STBC encoding is designed to enhance the performance of MIMO channel by designing a $N_{t} x N_{\gamma}$ code matrix. The vector code contains the variables $S_{1}, S_{2 x^{* \cdots \cdots}} S_{\mathrm{m}}$ as mentioned in [9]. STBC is used with a block vise and therefore offers less relative benefits and other coding but due to orthogonal properties the performance is better in terms of error rate.

Space Time Block coded (STBC) - MIMO technique with OFDM is the most reliable way to obtain better bandwidth efficiency and ISI reduction capability. The BPSK modulation technique is believed to offer the better
BER performance over all other adaptive PSK based modulation schemes. Thus this paper proposed to use the BPSK for $2 x 1$ MIMO systems with STBC code. The received signals in the two respective channels time slots are

$$
\begin{aligned}
& r\left(t_{1}\right)=r_{1}=\left(h_{1} x_{1}+h_{2} x_{2}\right)+w_{1} \\
& r\left(t_{2}\right) \equiv T_{2} \equiv\left(-h_{1} x_{2}^{*}+h_{2} x_{1}^{*}\right)+w_{2}
\end{aligned}
$$

Where $\mathrm{h}$ represents the channel realization matrix and corresponds to CSI. The calculated minimum BPSK bit error rate in AWGN channel is mathematically given as

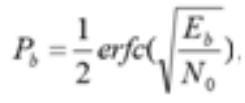

There may be certain methods which involve the feedback between the TX and RX for controlling the CSI information and realization. An Feedback example is shown in Figure 6.

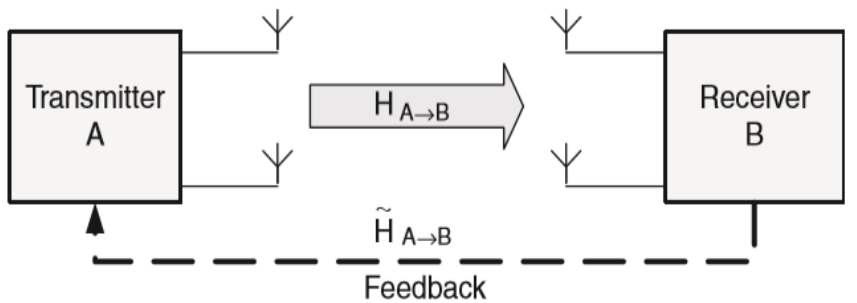

Fig.6. Feedback based CSI realization for MIMO STBC system.

The basic idea behind the STBC codes is to transfer a set of multiple copies of channel information to the recipient. This allows for a better survival rate if the information is in transit time. A variety of integrated error correction methods (FEC) can be used to continuously improve STBC performance. The basic architecture of the STBC based maximal rate channel combiner (MRC) based decoder is shown in the Figure 7.

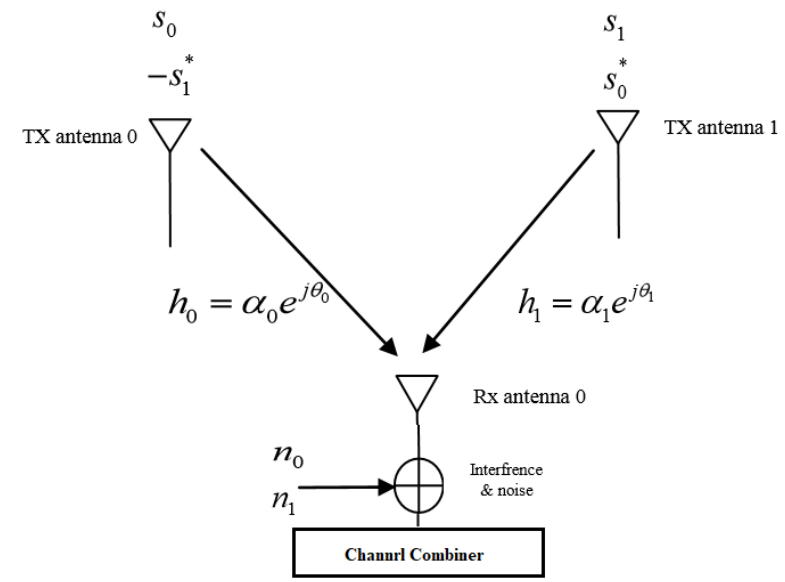

Fig.7. MRC based STBC -MIMO system

\section{CONCLUSION}

Primarily paper presets the study of the MIMO-STBC wireless system for the Wi-Fi routers. A classification of MIMO design based on the STBC coding is presented. The modeling of the MIMO channel using the OSTBC is presented. It is proposed to use the BPSK modulation for 
ISSN: 2277-3754

ISO 9001:2008 Certified

International Journal of Engineering and Innovative Technology (IJEIT)

Volume 11, Issue 4, October 2021

BER analysis as it offers better BER performance. Paper also addressed the basic design challenges in MIMO system. It is concluded the MIMO offers higher throughput and data capacity thus in the systems where small MIMO system are fusible, it s good to use the STBC coding for performance improvement. It is still a big challenge to design the system with the use of the space tie codes for performance enhancement.

\section{REFERENCES}

[1] S Nandi, A Nandi., "Performance Analysis of Alamouti STBC MIMO OFDM for Different Transceiver System", IEEE International Conference on Intelligent Sustainable Systems,pp:883-887,ICISS 2017.

[2] V. Tarokh, N. Seshadri, A.R. Calderbank, "Space-time codes for high data rate wireless communication: performance criterion and code construction", IEEE Transactions on Information Theory, vol. 44, issue. 2, pp: 744-765, 1998.

[3] Santumon. S. D, R. Sujatha, "Space-Time Block Coding (STBC) for Wireless Networks", International Journal of Distributed and Parallel Systems (IJDPS) vol.3, no.4, pp: 183-195, July 2012.

[4] S. Karri, R. Ramesh, G. Kiran, C. Manikanta and B. Gangadhar, "BER analysis of MIMO-Wimax system using orthogonal space time block codes," 2017 IEEE International Conference on Power, Control, Signals and Instrumentation Engineering (ICPCSI), pp. 30623068,2017 .

[5] J. P. Patra and P. Singh, "A comb-type pilot symbol aided channel estimation for STBC based OFDM system over frequency selective channel," 2013 IEEE Asia Pacific Conference on Postgraduate Research in Microelectronics and Electronics (Prime Asia), pp. 262-267. 2013.

[6] Manisha Patel, \& Tiwari, Rovin, "Pilot Assisted Channel Estimation in MIMO-STBC Systems over Time-Varying Fading Channels- A Review", International Journal of Computer Applications, Vol 151, pp: 23-27, 2016.

[7] Mohamed Marey, Octavia A. Dobre, Robert Inkol, "Blind STBC Identification for Multiple-Antenna OFDM Systems", IEEE Transactions On Communications, Vol. 62, No. 5, pp:1554-1567, May 2014.

[8] Kritika Gautam, and Pankaj Shukla, "Comparison of Space-Time Block Code under Different Fading channel", $2^{\text {nd }}$ IEEE International Conference on Communication and Electronics Systems, pp:234-239, 2017.

[9] P. K. M. Ho, K. Yar and P. Y. Kam, "Cutoff Rate of MIMO Systems in Rayleigh Fading Channels With Imperfect CSIR and Finite Frame Error Probability", IEEE Transactions on Vehicular Technology, Vol. 58, no. 7, pp. 3292-3300, Sept. 2009.

[10] Pankaj kumar bharti, Paresh Rawat, "Alamouti-STBC Based Performance Estimation of Multi $\mathrm{Tx}$ and $\mathrm{Rx}$ Antenna over MIMO-OFDM", $2^{\text {nd }}$ IEEE International conference on Trends in Electronics and informatics ICOEI, pp: 1277-1281, 2018.

[11] Aws Amer Kamil, Mahdi Abdul Hadi, "STBC-OFDM System under Rayleigh Fading Channel with Known and Unknown CSI at the transmitter", International Journal of
Innovative Research in Science, Engineering and Technology Vol. 7, Issue 5, May,pp:5377-5384, 2018.

[12] A. R. Trivedi, S. B. Parmar, S. B. Bhatt, "Comparison of different MIMO system-using STBC in Rayleigh channel", International Journal of Emerging Technology and Advanced Engineering, Volume 2, Issue 4, April 2012,pp:121-125,2012.

[13] M. Biguesh and A. B. Gershman, "Training-based MIMO channel estimation: A study of estimator tradeoffs and optimal training signals," IEEE Trans. Signal Process., vol. 54, no. 3, pp. 884-893, Mar. 2006. 